\title{
Risco cardiovascular, efetividade e mortalidade
}

Cardiovascular risk, effectiveness and mortality

Riesgo cardiovascular, efectividad y mortalidad

Juan Gérvas ${ }^{1}$

A Lei de Ferro da Epidemiologia (Ley de Hierro de La Epidemiología) diz que todos que nascem, morrem. Por isso, o propósito da Medicina não é evitar a morte por si só, mas evitar as mortes, as doenças e o sofrimento que podem ser medicamente evitáveis.

No final, todos nossos pacientes morrerão - e nós também, obviamente, morreremos. "Os corpos encontram uma forma de morrer", e se a causa não for por fome ou desidratação, por motivo congênito e infeccioso, por lesões, câncer ou suicídio, temos que esperar que seja por 'motivo cardiovascular', doença pulmonar, insuficiência renal ou hepática, demência ou outras doenças degenerativas. Mas temos que morrer por alguma coisa.

Morrer por causa cardiovascular não é desonroso, nem refere-se à atenção clínica imperfeita. $\mathrm{O}$ fato de a primeira causa de morte ser a cardiovascular não tem nenhuma relação com os cuidados clínicos e nem deveria assustar.

Entretanto, muitas das mortes por motivo cardiovascular poderiam ser evitadas. Assim, poder-se-ia evitar mortalidade cardiovascular, diminuindo a desigualdade social, por exemplo, com melhor redistribuição da riqueza, melhor educação etc. Os médicos sabem que os fatores adversos psicossociais associados ao fato de pertencer à classe baixa correspondem a $35 \%$ do risco atribuído à hipertensão na incidência do infarto do miocárdio (em outra hipótese, pertencer à classe baixa duplica 2,7 tal risco $)^{1}$.

Também deve-se saber que, contra as mortes cardiovasculares, não há nada como as políticas de saúde pública quanto ao tabagismo (restrições dos lugares onde fumar, aumento do preço do tabaco, campanhas de informação, entre outras).

$\mathrm{Na}$ parte clínica, as mortes cardiovasculares evitáveis devem ser vistas em perspectiva, de acordo com o que seja possível conseguir². Portanto, por 100.000 habitantes ao ano, o tratamento com inibidores da enzima conversora de angiotensina (IECA) na insuficiência cardíaca pode evitar 308 mortes; o breve conselho médico contra o tabaco, 120; o tratamento da hipertensão, 71; o uso de aspirina para o infarto do miocárdio, 48; o tratamento anticoagulante na fibrilação auricular, 33; o uso das estatinas para o infarto do miocárdio (prevenção secundária), 14; e o tratamento com estatinas nos pacientes com risco cardiovascular (prevenção primária), 3 mortes. Por isso, o uso das estatinas para prevenir mortes cardiovasculares em pacientes sem doenças coronárias é muito ineficiente.

Departamento de Saúde Pública, Faculdade de Medicina da Universidad Autónoma - Madrid, Espanha. jgervasc@meditex.es 
O risco cardiovascular é calculado seguindo tabelas adequadas. A mais conhecida é a de Framingham. Essa tabela foi obtida com dados de uma população anglo-saxã, que forneceram resultados muito diferentes de outras populações, como as mediterrâneas em que se aplica com melhor validade científica a tabela REGICOR ${ }^{3}$. Talvez, a tabela de Framingham também seria inapropriada para a população brasileira. Em todo caso, as tabelas de risco cardiovascular são aplicáveis às populações, não aos sujeitos.

As tabelas de risco não são tabelas de decisão. Ou seja, não permitem tomar decisões na consulta com o paciente. Transformam-se em tabelas de decisão por meio da análise do impacto, que não foi feita com as tabelas de risco cardiovascular ${ }^{4}$.

Portanto, passar da população (tabela de risco) ao paciente na consulta é uma tragédia 'clínica-estatística's.

Assim, se os pacientes forem acompanhados por dez anos, a incidência dos infartos do miocárdio será muito menor do que a esperada nos pacientes classificados como de alto risco (e será muito maior do que a prevista nos de 'baixo risco') ${ }^{6}$.

Há um excesso, muito risco cardiovascular na mesma proporção das mortes cardiovasculares ${ }^{7}$. As tabelas de risco são inúteis e perigosas para se aplicar a um paciente individualmente $\mathrm{e}^{4,8}$.

As tabelas de risco cardiovascular deveriam ser retiradas da consulta ao médico para proteger o paciente dos excessos no diagnóstico e no tratamento.

Os médicos podem se confundir ao atribuírem aos fatores de risco um componente causal. Os fatores de risco não são causa, mas sim associações estatísticas. Os fatores de risco não são nem causa, nem são necessários ou suficientes?.

As estatísticas do fator de risco nos pacientes dizem pouco a respeito do problema da mortalidade cardiovascular na população. É mais conveniente observar as estatísticas na perspectiva e não empregá-las para justificar o uso inadequado das estatinas e de outras terapêuticas. Os fatores de risco podem levar à confusão ${ }^{10}$.

A prevenção primária cardiovascular com estatinas não tem fundamento científico ${ }^{11}$.

Lamentavelmente, os médicos empregam as estatinas em excesso para a prevenção primária - na qual são inúteis e perigosas - e pouco na prevenção secundária - quando há isquemia coronária, sendo úteis e necessárias ${ }^{12,13}$.

O mais lógico seria se concentrar na prevenção secundária como clínicos e deixar a prevenção primária cardiovascular para o trabalho conjunto dos médicos gerais/de família com a saúde pública (saúde coletiva), por exemplo, contra o tabagismo, oferecendo o melhor de ambos os mundos para a sociedade.

\section{Referências}

1. Lang T. Ignoring social factors in clinical decision rules: a contribution to health inequalities? Eur J Public Health. 2005; 15(5): 441. http://dx.doi.org/10.1093/eurpub/cki156

2. Fleetcroft R, Cookson R. Do the incentive payments in the new NHS contract for primary care reflect likely population health gaines? J Health Serv Res Policy. 2006; 11(1): 27-31. http://dx.doi.org/10.1258/135581906775094316

3. Miguel F, García A, Montero MJ. Prevención primaria con estatinas, consensos, y tablas de riesgo. Aten Primaria. 2005; 36: 31-8. http://dx.doi.org/10.1157/13075929

4. Reilly BM, Evans AT. Translating clinical research into clinical practice. Impact of using prediction rules to make diagnosis. Ann Intern Med. 2006; 144(3): 201-9.

5. Feinstein AR. The problem of cogent subgroups: a clinicostatistical tragedy. J Clin Epidemiol. 1998; 51(4): $297-9$. http://dx.doi.org/10.1016/S0895-4356(98)00004-3

6. Jimeno J, Molist N, Franch J, Serrano V, Serrano L, Gracia R. Variabilidad en la estimación del riesgo coronario en diabetes mellitus tipo 2. Aten Primaria. 2005; 35(1): 30-6. http://dx.doi.org/10.1157/13071042

7. Betancor León P. [Demasiado riesgo cardiovascular y enfermedad poco frecuente]. Med Clin (Barc). 2000; 115(1): 738-9. Spanish.

8. Gérvas J, Pérez Fernández M. Dislipemias. En: Uso Racional del Medicamento. Coordinador L.M. García Olmos. Madrid: Ars XXI; 2007. [curso a distancia] http://www.equipocesca.org/uso-apropiado-de-recursos/dislipemias/ 
9. Miguel García F. [Factores de riesgo: una nada inocente ambigüedad en el corazón de la medicina actual]. Aten Primaria. 1998; 22(9): 585-95. Spanish.

10. Starfield B, Hyde J, Gérvas J, Heath I. The concept of prevention: a good idea gone astray? J Epidemiol Community Health. 2008; 62(7): 580-3. http://dx.doi.org/10.1136/jech.2007.071027

11. ¿Tienen algún papel las estatinas en prevención primaria?. Actualización de la evidencia. Therapeutics Initiative Letter. [Internet]. 2010; 77. [cited 2011 Dec 5]. Available from: http://www.ti.ubc.ca/es/newsletter/\%C2\%BFtienenalg\%C3\%BAn-papel-las-estatinas-en-prevenci\%C3\%B3n-primaria-actualizaci\%C3\%B3n-de-la-evidencia.

12. Maiques Galán A, Villar Alvarez F, Llor Vila C, Torcal Laguna J. El riesgo coronario en España y el tratamiento con fármacos hipolipemiantes. Aten Primaria. 2003; 32(7): 420-2. Spanish.

13. Ray KK, Seshasai SR, Erqous S, Sever P, Jukema JW, Ford I, et al. Statins and all-causes mortality in high risk primary prevention. Arch Intern Med. 2010; 170(12): 1024-31. http://dx.doi.org/10.1001/archinternmed.2010.182 